\title{
Elecsys CSF biomarker immunoassays demonstrate concordance with amyloid- PET imaging
}

James D. Doecke ${ }^{1,2^{*}}$, Larry Ward', Samantha C. Burnham³, Victor L. Villemagne ${ }^{4,5}$, Qiao-Xin Li ${ }^{4}$, Steven Collins ${ }^{4,6}$, Christopher J. Fowler ${ }^{4}$, Ekaterina Manuilova ${ }^{7}$, Monika Widmann ${ }^{8}$, Stephanie R. Rainey-Smith ${ }^{9}$, Ralph N. Martins ${ }^{10,11}$, Colin L. Masters ${ }^{4}$ and the AIBL Research Group

\begin{abstract}
Background: $\beta$-amyloid (A $\beta$ ) positron emission tomography (PET) imaging is currently the only Food and Drug Administration-approved method to support clinical diagnosis of Alzheimer's disease (AD). However, numerous research studies support the use of cerebrospinal fluid (CSF) biomarkers, as a cost-efficient, quick and equally valid method to define AD pathology.

Methods: Using automated Elecsys ${ }^{\circledast}$ assays (Roche Diagnostics) for $A \beta(1-42)(A \beta 42), A \beta(1-40)(A \beta 40)$, total tau (tTau) and phosphorylated tau (181P) (pTau), we examined CSF samples from 202 participants of the Australian Imaging, Biomarkers and Lifestyle (AIBL) study of ageing cohort, to demonstrate the concordance with pathological $A D$ via PET imaging.

Results: Ratios $A \beta 42 / A \beta 40, \mathrm{tTau} / \mathrm{A} \beta 42$ and $\mathrm{pTau} / \mathrm{A} \beta 42$ had higher receiver operator characteristic - area under the curve (all 0.94), and greater concordance with Aß-PET (overall percentage agreement $~ 90 \%$ ), compared with individual biomarkers.

Conclusion: Strong concordance between CSF biomarkers and AB-PET status was observed overall, including for cognitively normal participants, further strengthening the association between these markers of $A D$ neuropathological burden for both developmental research studies and for use in clinical trials.
\end{abstract}

Keywords: Alzheimer's disease, Beta-amyloid, Cerebrospinal fluid, Concordance PET, Tau

\section{Background}

Alzheimer's disease $(\mathrm{AD})$ pathology is now recognised to evolve over an extended period before the onset of clinical symptoms [1], with homeostatic failure of the amyloid precursor protein cleavage appearing to be the primary pathogenic event [2-4]. The resulting accumulation of $\beta$-amyloid (A $\beta$ ) peptides into senile plaques is coupled with the

\footnotetext{
* Correspondence: james.doecke@csiro.au

'Cooperative Research Council for Mental Health, Melbourne, Victoria 3052, Australia

${ }^{2}$ Australian E-Health Research Centre, CSIRO Health \& Biosecurity, Level 5, 901/ 16 Royal Brisbane \& Women's Hospital, Brisbane, Queensland 4029, Australia Full list of author information is available at the end of the article
}

degeneration of neurons, abnormal hyperphosphorylation of the tau protein and formation of tau neurofibrillary tangles $[5,6]$. To date, visual assessment of $A \beta$ positron emission tomography (PET) scans is the only Food and Drug Administration-approved method to support the clinical diagnosis of $\mathrm{AD}$ [7]. Whilst measurement of neocortical amyloid via PET is recognised as a core marker of disease pathological status, A $\beta$-PET imaging is costly and is not easily amenable for application to the wider community. Inclusion of cerebrospinal fluid (CSF), total tau (tTau) and phosphorylated tau (181P) (pTau) in ratios with $A \beta$ (1-42) (A $\beta 42)$ has been shown to improve biomarker 
performance, reflecting the strong relationship between the presence of both $\mathrm{A} \beta$ and tau pathologies in $\mathrm{AD}[8$, 9]. Recent studies have shown good concordance between $\mathrm{A} \beta 42$ levels, and tTau/A $\beta 42$ and $\mathrm{pTau} / \mathrm{A} \beta 42$ ratios measured in CSF using a variety of platforms, including automated Elecsys ${ }^{\bullet}$ assays (Roche Diagnostics), and $\mathrm{A} \beta$-PET outcome obtained using different radiotracers in diverse study cohorts [10, 11]. Furthermore, CSF biomarker status determined using predefined thresholds has been shown to predict clinical decline and progression to dementia in patients with mild cognitive impairment (MCI) $[10,12]$. Accordingly, the use of CSF biomarkers to support AD diagnosis is recommended in recent research diagnostic guidelines published by the International Work Group 2 [13], as well as the National Institute on Ageing-Alzheimer's Association (NIA-AA) biological framework for AD [14].

The present study aimed to provide additional evidence in support of the relationship between CSF biomarkers and the neuropathological A $\beta$-PET classification, thus further contributing to the NIA-AA research framework, which utilises the measurement of biomarkers to define an $\mathrm{AD}$ continuum $[14,15]$. The relationship between CSF biomarkers $A \beta 42, A \beta$ (1-40) $(\mathrm{A} \beta 40)$, $\mathrm{tTau}$ and $\mathrm{pTau}$ (and their ratios) with the neuropathological A $\beta$-PET classification status was evaluated both across the full clinical disease spectrum, and in cognitively normal controls from the highly characterised Australian Imaging, Biomarkers and Lifestyle (AIBL) study of ageing cohort.

\section{Methods}

\section{Sample collection}

This was a retrospective analysis of a sub-cohort of 202 participants from the AIBL study of ageing cohort [16], which utilised CSF samples and available neuropathological $A \beta$ imaging data. Samples were included from participants who were diagnosed as either cognitively normal (CN; $n=140)$, with MCI (not necessarily due to $\mathrm{AD}, n=33)$, with $\mathrm{AD}(n=27)$ or with frontotemporal dementia (FTD; $n=2$ ) via a cognitive and a subjective neuropsychological assessment. Prior written informed consent was obtained from all participants, and ethical approval was provided by all participating institutions.

\section{CSF collection}

The CSF collection protocol has been published previously $[17,18]$ and is aligned with the Alzheimer's Biomarkers Standardization Initiative [19]. Following an overnight fast, CSF was collected in the morning by lumbar puncture using a Temena (Polymedic ${ }^{\circ}, \mathrm{EU}$ ) spinal needle micro-tip $(22 / 27 \mathrm{G} \times 103 \mathrm{~mm}$; CAT 2192227). Aseptic technique was adhered to at all times, with the participants sitting upright. CSF was collected by either gravity or aspiration into 15-mL polypropylene tubes (Greiner Bio-One188271). Samples were placed on ice immediately and kept between $2{ }^{\circ} \mathrm{C}$ and $8{ }^{\circ} \mathrm{C}$ during transport to the laboratory, and processed within $1 \mathrm{~h}$. Samples were centrifuged at $2000 \times g$, at $4{ }^{\circ} \mathrm{C}$ for $10 \mathrm{~min}$ and supernatant transferred to a fresh Greiner polypropylene tube and gently inverted. Aliquots were snapfrozen in 1-mL screw-cap 2D barcoded polypropylene Nunc Cryotubes (NUN374088) for long-term storage; samples were stored in liquid nitrogen vapour tanks until use and thawed once immediately before analysis.

\section{Immunoassays}

The Elecsys $\beta$-Amyloid (1-42) CSF, Elecsys $\beta$-Amyloid (1-40) CSF, Elecsys Total Tau CSF and Elecsys Phospho-Tau (181P) CSF assays are electrochemiluminescence immunoassays, which can be run on cobas e 601, cobas e 602 and MODULAR ANALYTICS E170 analysers. The assays have measuring ranges of 200$1700 \mathrm{pg} / \mathrm{mL}$ (A $\beta 42), 0.011-39.540 \mathrm{ng} / \mathrm{mL}$ (A $\beta 40), 80$ $1300 \mathrm{pg} / \mathrm{mL}$ (tTau) and $8-120 \mathrm{pg} / \mathrm{mL}$ (pTau). The Elecsys $\beta$-Amyloid (1-40) assay is currently employed for research use only. Further information on each assay, including standardisation and analytical performance, can be found in previous publications [20-22]. Of the CSF biomarkers that were measured, $22 \%$ of the observations for $A \beta 42$ were above the upper limit of the assay measuring range. Further information regarding how this was dealt with is shown in Additional file 1: Supplementary Methods.

\section{Amyloid-PET measurement}

A $\beta$-PET imaging was performed with four different radiotracers: ${ }^{11} \mathrm{C}$-Pittsburgh compound $\mathrm{B}(\mathrm{PiB})$, ${ }^{18}$ F-NAV4694 (NAV), ${ }^{18}$ F-Flutemetamol (FLUTE) or ${ }^{18} \mathrm{~F}$-Florbetapir (FBP). Methodology for each tracer has been previously described [23]. Briefly, standardised uptake values (SUVs) were calculated via summing spatially normalised PET images sampled using a narrow cortical regions of interest template (reducing possible noise from the measurement). The SUVs were then scaled to each tracer's recommended reference regions to define the SUV ratio (SUVR). Reference region for NAV and PiB was the cerebellar cortex [24, 25], for FLUTE the pons [26] and for FBP the whole cerebellum [27]. Given that data from the NAV and PiB tracers have almost identical dynamic ranges, and only one participant had measurements from NAV alone, data from these tracers were combined and labelled as "NAV/PiB". Quantitative SUVR values were dichotomised into A $\beta$-PET - or A $\beta$-PET+ based on each tracer-specific threshold (NAV/PiB: 1.4, FLUTE: 0.62 and FBP: 1.05). Briefly, for NAV/PiB, the binary A $\beta$-PET threshold was computed using a cluster analyses and compared with thresholds previously identified by 
Clark et al. [28], for FLUTE, Thurfjell et al. used a ROC method compared with post mortem results [29], and lastly for FBP, Clark et al. calculated the threshold to be the 95th percentile of the SUVR from young healthy controls (age 35-55 years and without cognitive impairment) [28].

\section{Population demographic comparisons}

Population demographic characteristics (gender, age, apolipoprotein E [APOE] $\varepsilon 4$ allele status, cognitive scores [the preclinical Alzheimer's cognitive composite (PACC), Mini-Mental State Examination (MMSE) and Clinical Dementia Rating (CDR)], PET tracer frequency and clinical classification/diagnoses) were compared in $\mathrm{A} \beta$-PET - and A $\beta$-PET + groups using chi-squared test, independent-samples $t$-test and Mann-Whitney $U$ test where appropriate.

\section{Comparisons of CSF biomarker means}

Distribution of CSF biomarkers in groups with different PET status were compared using the Wilcoxon signedrank test and generalised linear models accounting for covariates, including age, APOE $\varepsilon 4$ allele status, gender and clinical classification/diagnosis. For the biomarker comparisons between A $\beta$-PET status, the two participants with FTD (and ultimately not on an AD pathway) were not included in statistical analyses.

\section{Biomarker threshold construction}

Biomarker (both individual and ratio) thresholds were derived using the optimisation of Youden's index [30] within receiver operating characteristic-area under the curve (ROC-AUC) analyses using dichotomised A $\beta$-PET status as an endpoint. In addition, for $A \beta 42 / A \beta 40$, $\mathrm{pTau} / \mathrm{A} \beta 42$ and tTau/A $\beta 42$, which had clear bi-modal distributions, unsupervised thresholds were derived using two-component Gaussian mixture models (GMMs). Further information on the construction of the GMM's and the derivation of their thresholds is shown in Additional file 1: Supplementary Methods.

\section{Concordance and performance of the CSF biomarkers with A $\beta$-PET}

Elecsys CSF assay biomarkers and their respective ratios (A $442 / A \beta 40, t T a u / A \beta 42$ and $p T a u / A \beta 42)$ were analysed with respect to their concordance with $\mathrm{A} \beta$-PET status, irrespective of clinical classification, and within sample with cognitively normal participants. The capability of individual CSF biomarkers and various ratios to distinguish participants classified as $\mathrm{A} \beta-\mathrm{PET}+/-$ was assessed using ROC-AUC analyses. AUC values of individual biomarkers and biomarker ratios were compared using DeLong's method [31]. Overall, positive and negative percentage agreements (OPA, PPA and NPA, respectively) with A $\beta$-PET status were calculated at all derived thresholds.

\section{Results \\ Sample demographics and biomarker group-wise comparisons}

Ninety participants were imaged using the NAV/PiB tracer, 70 with the FLUTE tracer and 42 with the FBP tracer; more participants were $\mathrm{A} \beta$-PET - than $\mathrm{A} \beta$-PET+ with each tracer. Overall, 38/140 (27\%) CN participants, 23/33 (70\%) participants with MCI and 23/27 (85\%) participants with clinically diagnosed $\mathrm{AD}$ had $\mathrm{A} \beta$-PET+ (Table 1). Both participants with FTD were A $\beta$-PET-. Participants who were A $\beta$-PET + were more likely to be male $(P=0.03)$, older $(P=0.01)$ and be APOE $\varepsilon 4$ allele status positive $(P<0.0001)$, with poorer cognitive scores $(P<0.0003)$. All CSF biomarker means and medians were significantly different between $\mathrm{A} \beta$-PET groups $(P<0.0001$; Additional file 2: Supplementary Table S1), with distributional differences between pathological and clinical subgroups shown in Fig. 1.

\section{CSF biomarker thresholds}

Thresholds developed using the optimisation of Youden's index based on the complete cohort for individual biomarkers $A \beta 42$, tTau and pTau, and ratios $A \beta 42 /$ $\mathrm{A} \beta 40, \mathrm{tTau} / \mathrm{A} \beta 42$ and $\mathrm{pTau} / \mathrm{A} \beta 42$, were derived as 1054 $\mathrm{pg} / \mathrm{mL}, 213 \mathrm{pg} / \mathrm{mL}, 21.3 \mathrm{pg} / \mathrm{mL}$, and $0.064,0.258$ and 0.0183 , respectively. GMM analysis for the ratios resulted in the following thresholds: 0.0673 (95\% confidence interval $[\mathrm{CI}]$ 0.0612-0.0798) for $\mathrm{A} \beta 42 / \mathrm{A} \beta 40,0.165$ (95\% CI $0.150-0.187)$ for tTau/A 442 and 0.0159 (95\% CI 0.0141-0.0184) for pTau/A $\beta 42$. Biomarker distribution and goodness of fit are shown in Additional file 3: Supplementary Fig. S1 and Additional file 4: Supplementary Fig. S2.

\section{Concordance between CSF biomarkers and dichotomised A $\beta$-PET}

A 342 had the highest AUC among single biomarkers (0.86), followed by pTau (0.84) and tTau (0.81) (Fig. 2, Table 2). Compared with individual biomarkers, the ratios $\mathrm{A} \beta 42 / \mathrm{A} \beta 40$, tTau/A $\beta 42$ and pTau/A $\beta 42$ demonstrated a considerably higher performance $(P<0.0001)$, which was similar for all ratios (AUC of 0.94).

Among the single biomarkers, $A \beta 42$ had the highest concordance with $\mathrm{A} \beta$-PET status at the threshold optimised using Youden's index (OPA, PPA and NPA 81\%, Table 2). $A \beta 42 / A \beta 40$ and $p T a u / A \beta 42$ ratios outperformed single biomarkers and showed similar performance at the derived thresholds (OPA was 90\%, with a PPA and an NPA close to 90\%). Overall agreement to $\mathrm{A} \beta$-PET status using unsupervised thresholds was similar for $A \beta 42 / A \beta 40$ (90\%), and slightly lower for pTau/ 
Table 1 Study population demographic characteristics, including comparisons between A -PET groups

\begin{tabular}{|c|c|c|c|c|}
\hline Characteristic & Total sample & Aß-PET- & Aß-PET+ & $P$ value \\
\hline$\overline{n(\%)}$ & $202(100)$ & $118(58)$ & $84(42)$ & - \\
\hline Gender male, $n(\%)$ & $100(50)$ & $51(43)$ & $49(58)$ & 0.0340 \\
\hline Mean (SD) age, years & $73.5(6.2)$ & $72.5(6.2)$ & $74.8(6.0)$ & 0.0110 \\
\hline APOE $\varepsilon 4$ allele status carriage, $n(\%)$ & $64(32)$ & $24(21)$ & $40(48)$ & $<0.0001$ \\
\hline Mean (SD) PACC score & $-3.0(6.8)$ & $-0.5(4.2)$ & $-6.8(8.1)$ & $<0.0001$ \\
\hline Median (IQR) MMSE score & $28(4.0)$ & $29(2.0)$ & $27(4.2)$ & 0.0002 \\
\hline Median (IQR) CDR score & $0(2.4)$ & $0(0)$ & $0.5(3.2)$ & 0.0002 \\
\hline Tracer, $n(\%)$ & & & & 0.048 \\
\hline $\mathrm{NAV} / \mathrm{PiB}$ & $90(44)$ & $46(23)$ & $44(22)$ & - \\
\hline FLUTE & $70(35)$ & $41(20)$ & $29(14)$ & - \\
\hline FBP & $42(21)$ & $31(15)$ & $11(6)$ & - \\
\hline Clinical classification, n (\%) & & & & $<0.0001$ \\
\hline$C N$ & $140(70)$ & $102(51)$ & $38(19)$ & - \\
\hline $\mathrm{MCl}$ & $33(16)$ & $10(5)$ & $23(11)$ & - \\
\hline$A D$ & $27(13)$ & $4(2)$ & $23(11)$ & - \\
\hline FTD & $2(1)$ & $2(1)$ & $0(0)$ & - \\
\hline
\end{tabular}

Abbreviations: $A \beta, \beta$-amyloid; $A D$, Alzheimer's disease; $A P O E$, apolipoprotein $\mathrm{E} ; C D R$, Clinical Dementia Rating; $C N$, cognitively normal; $F B P,{ }^{18} \mathrm{~F}$-florbetapir; $F L U T E$,

${ }^{18}$ F-flutemetamol; $F T D$, frontotemporal dementia; IQR, interquartile range; $M C l$, mild cognitive impairment; MMSE, Mini-Mental State Examination; NAV,

${ }^{18}$ F-NAV4694; PACC, Preclinical Alzheimer Cognitive Composite; PET, positron emission tomography; PiB, ${ }^{11} \mathrm{C}$-Pittsburgh compound B; SD, standard deviation

$\mathrm{A} \beta 42(86 \%)$ and $\mathrm{tTau} / \mathrm{A} \beta 42(85 \%)$ ratios than agreement when using thresholds derived by optimisation of Youden's index (Table 2). The unsupervised threshold values were higher for $A \beta 42 / A \beta 40$ and lower for the Tau/A $\beta 42$ ratios than the optimised thresholds, resulting in slightly higher PPA and lower NPA.

\section{Concordance between CSF biomarkers and SUVR}

We investigated the relationship between the insoluble aggregated form of $A \beta$ (via quantitative NAV/PiB SUVR) and the soluble form of $A \beta$ via CSF biomarkers $A \beta 42$, the $A \beta 42 / A \beta 40$ ratio and the $p T a u / A \beta 42$ ratio. Given the nature of the two different pools of $A \beta$, we performed non-linear regression to estimate the relationship. Using threshold lines for the CSF biomarker and SUVR, Fig. 3 shows high concordance between CSF and $A \beta$-PET status, and the specific relationships between SUVR and CSF biomarkers. Additional file 5: Supplementary Fig. S3 demonstrates qualitatively similar relationships for both FLUTE and FBP tracers.

\section{Correlation structure between CSF $A \beta 42$ and CSF tau}

Scatter plots for $A \beta 42$ versus tTau and $A \beta 42$ versus pTau showed two clusters (Fig. 4). The majority of A $\beta$ PET - participants had values aligning close to the $x$-axis, whilst those participants who were A $\beta$-PET+ had values aligning close to the $y$-axis (Fig. 4a, b). Diagonal lines corresponding to the thresholds derived by the optimisation of Youden's index for ratios tTau/A 342 (Fig. 4a) and pTau/Aß42 (Fig. 4b), clearly separating participants with positive and negative A $\beta$-PET status. Apparent clusters (red for A $\beta$-PET+ and blue for A $\beta$-PET-) demonstrated the ability of the marker to align with neuropathological amyloid load.

\section{PET concordance analysis split by tracer}

Given that four different tracers were used within this study and that the relationship between PET tracer and correlated biomarker has previously been shown to vary by tracer [32, 33], all binary PET comparisons were performed using tracer-specific PET status. Whilst the distribution of $\mathrm{A} \beta-\mathrm{PET}+/$ - participants was different between tracers, results of ROC-AUC analysis were similar (Additional file 6: Supplementary Table S2), with small differences in performance possibly due to the limited sample sizes.

\section{PET concordance analysis in $\mathrm{CN}$ samples}

Biomarker thresholds developed based on the optimisation of Youden's index were $1046 \mathrm{pg} / \mathrm{mL}$ for $\mathrm{A} \beta 42$, and 0.064, 0.184 and 0.0186 for $\mathrm{A} \beta 42 / \mathrm{A} \beta 40$, tTau/A $\beta 42$ and $\mathrm{pTau} / \mathrm{A} \beta 42$, respectively. Agreement OPA was $83 \%$ for $\mathrm{A} \beta 42$ and was $89 \%, 89 \%$ and $90 \%$ for $\mathrm{A} \beta 42 / \mathrm{A} \beta 40$, tTau/ $\mathrm{A} \beta 42$ and $\mathrm{pTau} / \mathrm{A} \beta 42$, respectively (Additional file 7: Supplementary Table S3). We observed a decrease in PPA and NPA of only $1 \%$ for $A \beta 42 / A \beta 40$ and pTau/A $\beta 42$ ratios, with very similar optimised threshold values compared with the whole population. The threshold for tTau/ A $\beta 42$ was slightly lower for $\mathrm{CN}$ participants, resulting in a higher PPA ( $92 \%$ vs $83 \%$ in the whole population) and lower NPA ( $87 \%$ vs $97 \%$ in the whole population). 


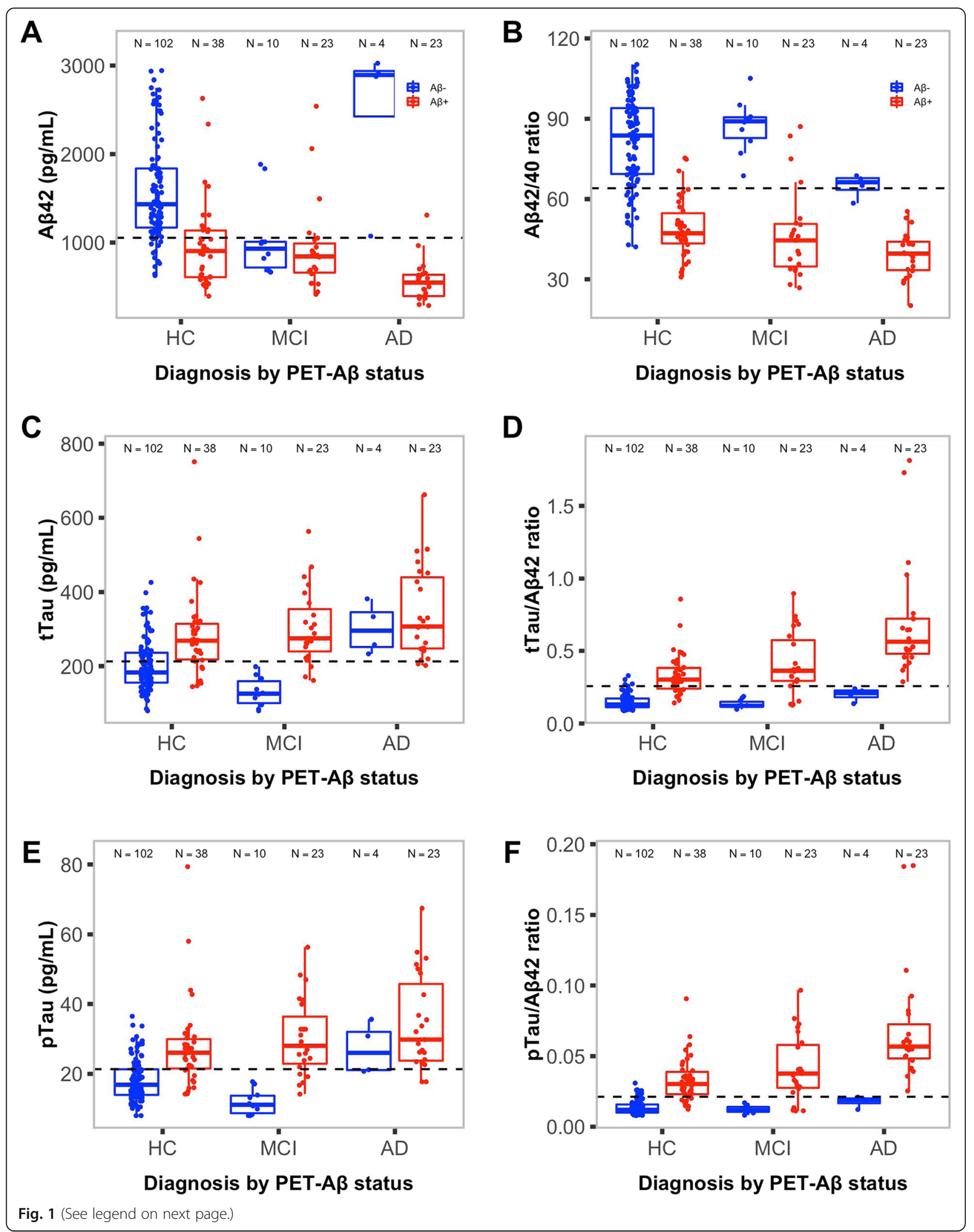


(See figure on previous page.)

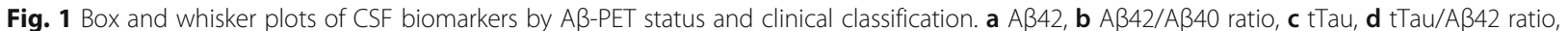
e pTau and $\mathbf{f}$ pTau/A 342 ratio. Dashed lines represent threshold values for each CSF biomarker as calculated via ROC analyses. Abbreviations: $A \beta$, $\beta$-amyloid; $A \beta 42, \beta$-amyloid (1-42); $A \beta 42 / A \beta 40, \beta$-amyloid (1-42)/ $\beta$-amyloid (1-40) ratio; $A D$, Alzheimer's disease; CSF, cerebrospinal fluid; HC, healthy controls; $M C$, mild cognitive impairment; PET, positron emission tomography; pTau, phosphorylated tau (181P); pTau/A 42 , phosphorylated tau (181P)/ $\beta$-amyloid (1-42) ratio; ROC, receiver operating characteristic; tTau, total tau; tTau/A 42 , total tau/ $\beta$-amyloid (1-42) ratio

\section{Discussion}

The current study shows high concordance between neuropathological AD CSF biomarkers and A $\beta$-PET classification in both the overall sub-cohort of AIBL, and in the subgroup of $\mathrm{CN}$ participants.

When comparing A $\beta$-PET concordance for the CSF biomarker ratios across the complete cohort, irrespective of clinical classification, the GMM unsupervised thresholds resulted in higher PPA and lower NPA than the ROC-AUC thresholds. The unsupervised thresholds distinguish AD-like from non-AD-like biomarker profiles. In our study, $90 \%$ of participants with $A D$-like CSF in the $A \beta 42 / A \beta 40$ ratio were $\mathrm{A} \beta-\mathrm{PET}+$, and $90 \%$ of participants with non-AD-like CSF in the $A \beta 42 / A \beta 40$ ratio were $A \beta-P E T-$. The corresponding numbers for the pTau/A $\beta 42$ ratio were $90 \%$ and $91 \%$, and for the tTau/A 342 ratio were $83 \%$ and $97 \%$. Only $3-10 \%$ of patients with non-AD-like biomarker profiles were $A \beta$ PET+, but $10-17 \%$ of patients with pathological CSF biomarker profiles were A $\beta$-PET-. This finding is consistent with the notion that CSF biomarkers are able to identify participants at risk of developing clinical $\mathrm{AD}$ much earlier than A $\beta$-PET. However, in our cohort, this notion could not be confirmed due to the very small number of participants whose diagnosis changed during the follow-up period.

CSF $\mathrm{Tau} / \mathrm{A} \beta 42$ and $\mathrm{A} \beta 42 / \mathrm{A} \beta 40$ ratios demonstrated greater concordance with $\mathrm{A} \beta$-PET status compared with individual biomarkers. In particular, OPA of the Tau/ $\mathrm{A} \beta 42$ (90\%) and $\mathrm{A} \beta 42 / \mathrm{A} \beta 40$ (90\%) ratios outperformed A $\beta 42$ alone (81\%). These results are consistent with previously published PET concordance studies using Elecsys assays. For example, concordance analysis with visual PET outcome in a subset of patients with mild cognitive symptoms from the Swedish BioFINDER cohort showed that the CSF tTau/A $\beta 42$ and $\mathrm{pTau} / \mathrm{A} \beta 42$ ratios have a higher OPA (90\%) compared with A $\beta 42$ alone (80\%) [10]. Similarly, in a Korean cohort of patients with AD, concordance with NAV/PiB, PET SUVR was improved using the $\mathrm{tTau} / \mathrm{A} \beta 42$ ratio (OPA, 92.5) over $\mathrm{A} \beta 42$ alone (OPA, 85.2\%) [34]. Consistent with these findings, a recent roadmap for $\mathrm{AD}$ biomarkers also identified the

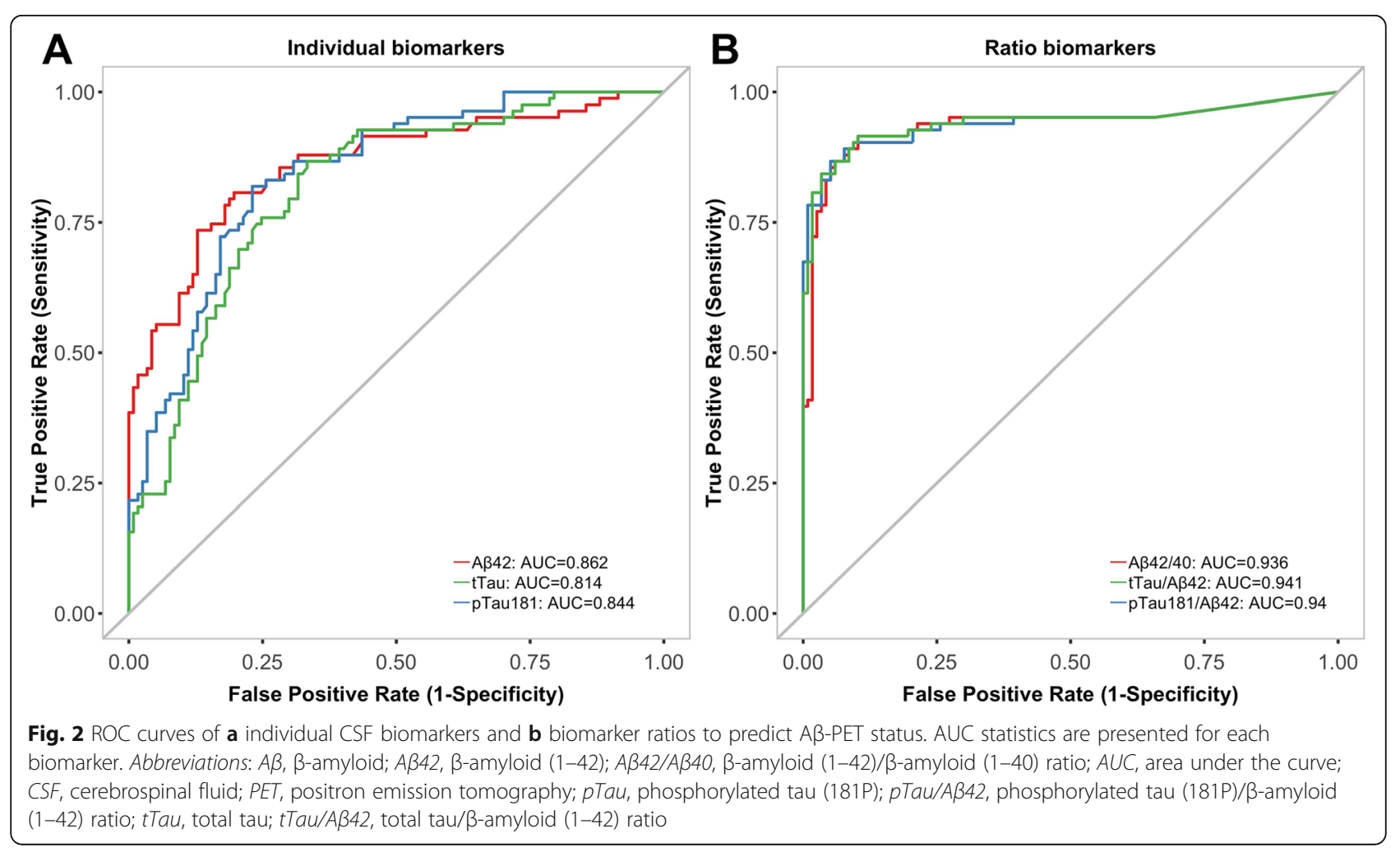


Table 2 ROC curve results-CSF biomarkers for prediction of Aß-PET status

\begin{tabular}{|c|c|c|c|c|c|c|}
\hline Biomarker & AUC (95\% Cl) & Threshold & Optimisation method & PPA (\%) & NPA (\%) & OPA (\%) \\
\hline$\overline{A \beta 42}$ & $0.86(0.81-0.92)$ & $1054 \mathrm{pg} / \mathrm{mL}$ & Youden & 81 & 81 & 81 \\
\hline tTau & $0.81(0.75-0.87)$ & $213 \mathrm{pg} / \mathrm{mL}$ & Youden & 86 & 66 & 75 \\
\hline pTau & $0.84(0.78-0.89)$ & $21.3 \mathrm{pg} / \mathrm{mL}$ & Youden & 81 & 77 & 79 \\
\hline \multirow[t]{2}{*}{$A \beta 42 / A \beta 40$} & $0.94(0.89-0.98)$ & 0.064 & Youden & 90 & 90 & 90 \\
\hline & & 0.0673 & GMM & 92 & 88 & 90 \\
\hline \multirow[t]{2}{*}{ tTau/Aß42 } & $0.94(0.90-0.98)$ & 0.258 & Youden & 83 & 97 & 91 \\
\hline & & 0.165 & GMM & 92 & 80 & 85 \\
\hline \multirow[t]{2}{*}{ pTau/Aß42 } & $0.94(0.90-0.98)$ & 0.0183 & Youden & 90 & 91 & 91 \\
\hline & & 0.0159 & GMM & 90 & 83 & 86 \\
\hline
\end{tabular}

Abbreviations: $A \beta$, $\beta$-amyloid; $A \beta 42, \beta$-amyloid (1-42); $A \beta 42 / A \beta 40, \beta$-amyloid (1-42)/ $\beta$-amyloid (1-40) ratio; $A U C$, area under the curve; $C l$, confidence interval; $C S F$, cerebrospinal fluid; GMM, Gaussian mixture model; $N P A$, negative percentage agreement; $O P A$, overall percentage agreement; $P E T$, positron emission tomography; $P P A$, positive percentage agreement; $p T a u$, phosphorylated tau (181P); $p$ Tau/A $\beta 42$, phosphorylated tau (181P)/ $\beta$-amyloid (1-42) ratio; ROC, receiver operating characteristic; tTau, total tau; tTau/Aß42, total tau/ $\beta$-amyloid (1-42) ratio

greater diagnostic utility of CSF biomarker ratios [15]. Possible explanations for the better performance of CSF biomarker ratios over $A \beta 42$ alone have been discussed previously [10]. Briefly, Tau/A $\beta 42$ ratios combine the two core biomarkers of the principal pathological processes, underlying $\mathrm{AD}$ into a single marker; combining measurements of two different proteins may compensate for natural fluctuations in the levels of each protein; and the temporal profile of $A \beta 42$ and tau biomarkers differ, with $A \beta 42$ considered to be an earlier metric of disease development than tau [35].

Similar to results from Schindler et al. [11], in cognitively normal patients assessed using PET with a cut-off of 1.42, performance of the pTau and tTau ratios with
A $\beta 42$ was very close to that of the $A \beta 42 / 40$ ratio (OPA AIBL A $\beta 42 / 40$ : 90\%, OPA ADRC A $\beta 42 / 40$ : 86\%; OPA AIBL pTau/A $342:$ 91\%, OPA ADRC pTau/A $342: 89 \%$; OPA AIBL tTau/Aß42: 91\%, OPA ADRC tTau/Aß42: $87 \%)$. Given the stage at which a participant is measured as $\mathrm{A} \beta-\mathrm{PET}+$, it is likely that the amyloid accumulation has caused synaptic damage, causing Tau to be released and accumulate into tangles (as a secondary event). As such the CSF Tau biomarkers are increasing and the CSF A $\beta 42$ is simultaneously decreasing.

Given the inherent relationship between CSF biomarkers and $A \beta$-PET status, we investigated the relationship between NAV/PiB SUVR and the CSF biomarkers $A \beta 42, A \beta 42 / A \beta 40$ ratio, $\mathrm{tTau} / \mathrm{A} \beta 42$ ratio and $\mathrm{pTau} / \mathrm{A} \beta 42$ ratio. The Tau/A $\beta 42$
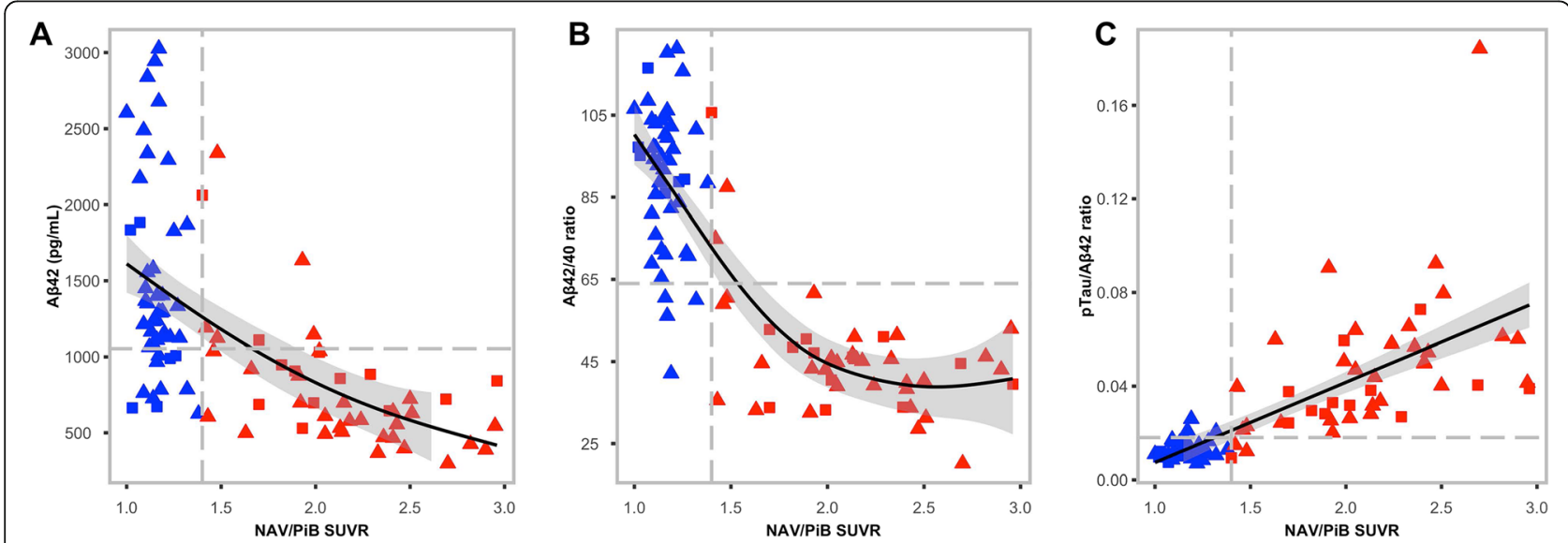

Fig. 3 CSF biomarkers versus NAV/PiB SUVR for a A 442 , threshold: 1054, b A $42 / A \beta 40$ ratio, threshold: $64.0(\times 0.001)$ and c pTau/A 342 ratio,threshold: 0.018 . Solid lines in plots $\mathbf{a}$ and $\mathbf{b}$ represent the non-linear relationship between CSF biomarkers and NAV/PiB SUVR. The solid line in plot c represents the linear relationship between NAV/PiB SUVR and pTau/Aß42. Grey shaded areas represent the $95 \% \mathrm{Cl}$ around the solid line. Grey dashed lines represent thresholds for SUVR (vertical) and CSF (horizontal) biomarkers. Red symbols represent Aß-PET+; blue symbols represent $A \beta-P E T-$; circles represent $C N$ participants; triangles represent participants with $M C l$; squares represent participants with $A D$. Abbreviations: $A \beta, \beta$-amyloid; $A \beta 42, \beta$-amyloid (1-42); $A \beta 42 / A \beta 40, \beta$-amyloid (1-42)/ $\beta$-amyloid (1-40) ratio; $A D$, Alzheimer's disease; $C l$, confidence interval; $C N$, cognitively normal; CSF, cerebrospinal fluid; $M C l$, mild cognitive impairment; NAV, ${ }^{18} \mathrm{~F}-\mathrm{NAV} 4694$; $P E T$, positron emission tomography; PiB, ${ }^{11}$ C-Pittsburgh compound B; pTau/Aß42, phosphorylated tau (181P)/B-amyloid (1-42) ratio; SUVR, standardised uptake value ratio 

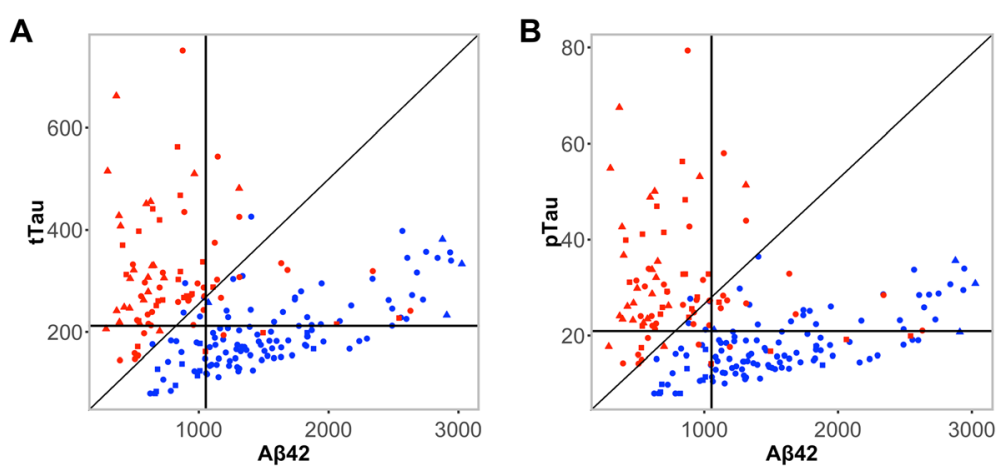

Fig. 4 CSF biomarker relationships for a tTau versus $A \beta 42$ and $\mathbf{b}$ pTau versus $A \beta 42$. Diagonal lines represent the split between the two clusters. Horizontal and vertical lines represent the cut-offs. Red symbols represent A $\beta-P E T+;$ blue symbols represent A $\beta$-PET-; circles represent cognitively normal participants; triangles represent participants with $\mathrm{MCl}$; squares represent participants with AD. Abbreviations: $A \beta, \beta$-amyloid; $A \beta 42, \beta$ amyloid (1-42); AD, Alzheimer's disease; CSF, cerebrospinal fluid; MCI, mild cognitive impairment; PET, positron emission tomography; pTau, phosphorylated tau (181P); tTau, total tau

ratios demonstrated a slightly higher overall agreement than the $A \beta 42 / A \beta 40$ ratio, albeit not significant, and both ratios outperformed A $\beta 42$ alone. Similar relationships were observed using FLUTE and FBP tracers.

Good concordance between CSF biomarkers and A $\beta$ PET classification was also observed in the subset of participants with normal cognition, and the degree of concordance was equivalent to that observed in the whole study population, differing only by approximately $1 \%$ in terms of NPA and PPA for the majority of biomarkers. These results support the opinion that $\mathrm{AD}$ pathogenesis is progressive and continuous, and changes in biomarkers occur prior to the onset of clinical symptoms. Our findings in CSF samples from AIBL study participants are consistent with those previously reported in other clinical cohorts, including BioFINDER and Alzheimer's Disease Neuroimaging Initiative (ADNI). Given the large proportion of cognitively normal elderly in the AIBL population, as compared with BioFINDER and ADNI, which had larger sample sizes in their $\mathrm{MCI}$ and $\mathrm{AD}$ populations, it is interesting that similar results overall were identified across these groups. This adds to the current knowledge that these neuropathological CSF biomarkers are highly predictive of amyloid plaques, irrespective of clinical stage.

This study presents research-based thresholds for CSF biomarkers for the separation of A $\beta$-PET groups similar to that of Hansson et al. [10] and Schindler et al. [11]; however, due to differences in sample handling and preanalytical procedures, which may potentially affect measured CSF biomarker levels and biomarker thresholds $[36,37]$, the thresholds are not directly comparable. Whilst this presents a problem for direct comparison of thresholds, the performance of this and other studies all point towards strong agreement between the soluble $A \beta$ and Tau as measured by CSF along with the measurement of the insoluble amyloid as measured by PET MRI.
Limitations of the present study include that PET SUVR is a proxy for histopathology, which is the current "gold standard" for establishing amyloid status. Additionally, the current research study used several radiotracers; this is however, reflective of both true clinical practice and research studies whereby funding constraints affect the ability to scan patients/participants. Reassuringly, results of the ROC-AUC analyses here were similar across the tracers. Retrospective samples were used from a small subset of participants, potentially reducing the reliability of our findings. The NPA and PPA values calculated at cut-offs derived by optimisation of Youden's index may be overoptimistic and should be validated in an independent data set.

A small number of participants who were diagnosed with AD-dementia or MCI did not have both tau and amyloid pathology (e.g. negative A $\beta$-PET, normal CSF $\mathrm{A} \beta 42$ and abnormal CSF tau levels), and thus neuropathological $\mathrm{AD}$ was likely not the cause of their cognitive impairment. Of interest, two participants with FTD were both $\mathrm{A} \beta$-PET-, demonstrating the absence of $\mathrm{AD}$ pathology in this type of dementia. As a strength, the study was therefore representative of a true population and shows the important role of biomarkers in differentiating $\mathrm{AD}$ from other forms of neurodegenerative diseases. Finally, the study is based on a single longitudinal research cohort, employing uniform approaches to all aspects, including CSF specimen handling, leaving some uncertainty about the generalisability of the findings to more diverse populations with a higher likelihood of less systematic technical rigour in relation to the biomarkers.

\section{Conclusion}

The AD CSF biomarkers showed high concordance with $\mathrm{A} \beta$-PET status in a cohort of individuals from the AIBL study. All three biomarker ratios (A $\beta 42 / A \beta 40$, tTau/A $\beta 42$ 
and $\mathrm{pTau} / \mathrm{A} \beta 42)$ demonstrated superior performance to A $\beta 42$ alone. These results further strengthen evidence supporting the potential diagnostic utility of CSF biomarkers, including the Elecsys platform biomarkers for identification of individuals at risk of $\mathrm{AD}$ in prodromal/ preclinical populations with normal cognition and early symptomatic patients, as well as for participant selection in therapeutic trials.

\section{Supplementary information}

Supplementary information accompanies this paper at https://doi.org/10. 1186/s13195-020-00595-5.

Additional file 1: Supplementary Methods. Information on handling of A 342 values above the measurement range and additional statistical information.

Additional file 2: Table S1. CSF biomarker univariate assessment with $A \beta$ status. Two participants with FTD were not included in statistical analyses.

Additional file 3: Figure S1. Threshold determination using mixture modelling for the biomarkers $(A, B) A \beta 42 / A \beta 40,(C, D) p T a u / A \beta 42$ and $(E$, F) tTau/Aß42.

Additional file 4: Figure S2. Threshold determination using mixture modelling for the biomarkers (A, B) A $442,(C, D)$ pTau and $(E, F)$ tTau.

Additional file 5: Figure S3. CSF biomarkers versus PET SUVR for: (A) $A \beta 42$ versus FLUTE SUVR, (B) A $342 / A \beta 40$ versus FLUTE SUVR, (C) pTau/ $A \beta 42$ versus FLUTE SUVR, (D) $A \beta 42$ versus FBP SUVR, (E) $A \beta 42 / A \beta 40$ versus FBP SUVR and (F) pTau/Aß42 versus FBP SUVR.

Additional file 6: Table S2. Results of ROC-AUC analysis - CSF biomarkers to predict A $\beta$-PET status, by PET tracer.

Additional file 7: Table S3. Results of ROC-AUC analysis - CSF biomarkers to predict AB-PET status in $\mathrm{CN}$ individuals.

\section{Abbreviations}

Aß: $\beta$-amyloid; AD: Alzheimer's disease; ADNI: Alzheimer's Disease Neuroimaging Initiative; AIBL: Australian Imaging, Biomarkers and Lifestyle; APOE: Apolipoprotein E; AUC: Area under the curve; CDR: Clinical Dementia Rating; Cl: Confidence interval; CN: Cognitively normal; CSF: Cerebrospinal fluid; FBP: ${ }^{18}$ F-Florbetapir; FLUTE: ${ }^{18}$ F-Flutemetamol; FTD: Frontotemporal dementia; GMM: Gaussian mixture model; HC: Healthy controls; IQR: Interquartile range; MCl: Mild cognitive impairment; MMSE: Mini-Mental State Examination; NAV: ${ }^{18}$ F-NAV4694; NIA-AA: National Institute on AgeingAlzheimer's Association; NPA: Negative percentage agreement; OPA: Overall percentage agreement; PACC: Preclinical Alzheimer's cognitive composite; PET: Positron emission tomography; PiB: ${ }^{11} \mathrm{C}-$ Pittsburgh compound B; PPA: Positive percentage agreement; pTau: Phosphorylated tau (181P); QQ: Quantile-quantile; ROC: Receiver operating characteristic; SD: Standard deviation; SUV: Standardised uptake value; SUVR: SUV ratio; tTau: Total tau; Aß40: $\beta$-amyloid (1-40); A 342 : $\beta$-amyloid (1-42)

\section{Acknowledgements}

We would like to thank the participants of the AIBL study, the members of $\mathrm{AIBL}$ and the team at CSIRO eHealth. Medical writing assistance, under the direction of the authors, was provided by David Evans, PhD, and Louise Kelly, BSc (Gardiner Caldwell Communications, Macclesfield, UK) and was funded by Roche Diagnostics. SC is an NHMRC Practitioner Fellow identification \#APP1105784. COBAS, COBAS E and ELECSYS are trademarks of Roche.

\section{Authors' contributions}

JDD contributed to the study design, data acquisition, data analyses, interpretation of results, writing of manuscript and critical review/revision of the manuscript; LW contributed to the study design, data acquisition, interpretation of results and critical review/revision of the manuscript; SCB contributed to the data acquisition, interpretation of results and critical review/revision of the manuscript; VLV contributed to the study design, data acquisition, interpretation of results and critical review/revision of the manuscript; QXL contributed to the study design, data acquisition, interpretation of results and critical review/revision of the manuscript; SC contributed to the study design, data acquisition, interpretation of results and critical review/revision of the manuscript; CJF contributed to the study design, data acquisition, interpretation of results and critical review/revision of the manuscript; EM contributed to the data analyses, interpretation of results and critical review/revision of the manuscript; MW contributed to the data analyses, interpretation of results and critical review/revision of the manuscript; SRRS contributed to the study design, data acquisition, interpretation of results and critical review/revision of the manuscript; RNM contributed to the study design, data acquisition, interpretation of results and critical review/revision of the manuscript; CLM contributed to the study design, data acquisition, interpretation of results and critical review/revision of the manuscript. The authors read and approved the final manuscript.

\section{Funding}

JDD received funding from CSIRO (AU). LW is funded by Cooperative Research Centre for Mental Health. SCB received funding from CSIRO (AU). SRRS is funded by the BrightFocus Foundation. The funding sources had no role in the study design, the collection, analyses, interpretation, writing of the report or the decision to submit the article for publication.

\section{Availability of data and materials}

Anonymised data is available upon request from any qualified investigator for the sole purpose of replicating procedures and results presented in the article.

\section{Ethics approval and consent to participate}

All procedures performed in studies involving human participants were in accordance with the ethical standards of the institutional and/or national research committee and with the 1964 Helsinki Declaration and its later amendments or comparable ethical standards. Written informed consent was obtained from all patients who participated in AIBL

\section{Consent for publication}

Not applicable.

\section{Competing interests}

JDD, LW, SCB, QXL, SC, CJF, SRRS, RNM and CLM declare that they have no competing interests. EM and MW are employees of Roche Diagnostics $\mathrm{GmbH}$. VLV reports speaker honoraria from GE Healthcare, Piramal Imaging and Avid Radiopharmaceuticals, and consulting fees from Lundbeck, AbbVie, Shanghai Green Valley Co and Hoffmann La Roche, all outside the scope of the submitted work.

\section{Author details}

${ }^{1}$ Cooperative Research Council for Mental Health, Melbourne, Victoria 3052, Australia. ${ }^{2}$ Australian E-Health Research Centre, CSIRO Health \& Biosecurity, Level 5, 901/16 Royal Brisbane \& Women's Hospital, Brisbane, Queensland 4029, Australia. ${ }^{3}$ Australian E-Health Research Centre, CSIRO, Parkville, Melbourne, Victoria 3052, Australia. 'The Florey Institute of Neuroscience and Mental Health, The University of Melbourne, Parkville, Melbourne, Victoria 3010, Australia. ${ }^{5}$ Department of Molecular Imaging and Therapy, Center for PET, Austin Health, Heidelberg, Victoria 3084, Australia. ${ }^{6}$ Department of Medicine (RMH), The University of Melbourne, Parkville, Melbourne, Victoria 3052, Australia. ${ }^{7}$ Roche Diagnostics GmbH, Nonnenwald 2, 82377 Penzberg, Germany. ${ }^{8}$ Roche Diagnostics GmbH, Sandhoferstrasse 116, 68305 Mannheim, Germany. ${ }^{9}$ Centre of Excellence for Alzheimer's Disease Research and Care, School of Medical and Health Sciences, Edith Cowan University, Joondalup, Western Australia 6027, Australia. ${ }^{10}$ Department of Biomedical Sciences, Macquarie University, North Ryde, New South Wales 2113, Australia. ${ }^{11}$ School of Psychiatry and Clinical Neurosciences, University of Western Australia, Crawley, Western Australia 6009, Australia.

Received: 11 October 2019 Accepted: 9 March 2020

Published online: 31 March 2020

\section{References}

1. Villemagne VL, Burnham S, Bourgeat P, Brown B, Ellis KA, Salvado O, et al. Australian Imaging Biomarkers and Lifestyle (AIBL) research group. Amyloid $\beta$ 
deposition, neurodegeneration, and cognitive decline in sporadic Alzheimer's disease: a prospective cohort study. Lancet Neurol. 2013;12:357-67.

2. Haass C, Selkoe DJ. Cellular processing of beta-amyloid precursor protein and the genesis of amyloid beta-peptide. Cell. 1993;75:1039-42.

3. Perneczky R, Alexopoulos P. Alzheimer's Disease Neuroimaging Initiative. Cerebrospinal fluid BACE1 activity and markers of amyloid precursor protein metabolism and axonal degeneration in Alzheimer's disease. Alzheimers Dement. 2014;10(Suppl):S425-9.e1.

4. Beason-Held LL, Goh JO, An Y, Kraut MA, O'Brien RJ, Ferrucci L, et al. Changes in brain function occur years before the onset of cognitive impairment. J Neurosci. 2013;33:18008-14.

5. Masters CL, Bateman R, Blennow K, Rowe CC, Sperling RA, Cummings JL. Alzheimer's disease. Nat Rev Dis Primers. 2015;1:15056.

6. Alzheimer's Association. 2017 Alzheimer's disease facts and figures. Alzheimers Dement. 2017:13:325-73.

7. Yang L, Rieves D, Ganley C. Brain amyloid imaging - FDA approval of florbetapir F18 injection. N Engl J Med. 2012;367:885-7.

8. Leuzy A, Chiotis K, Hasselbalch SG, Rinne JO, de Mendonça A, Otto M, et al. Pittsburgh compound B imaging and cerebrospinal fluid amyloid- $\beta$ in a multicentre European memory clinic study. Brain. 2016;139:2540-53.

9. Shaw LM, Waligorska T, Fields L, Korecka M, Figurski M, Trojanowski JQ, et al. Derivation of cutoffs for the Elecsys ${ }^{\circledast}$ amyloid $\beta$ (1-42) assay in Alzheimer's disease. Alzheimers Dement (Amst). 2018;10:698-705.

10. Hansson O, Seibyl J, Stomrud E, Zetterberg H, Trojanowski JQ, Bittner T, et al. Alzheimer's Disease Neuroimaging Initiative CSF biomarkers of Alzheimer's disease concord with amyloid- $\beta$ PET and predict clinical progression: a study of fully automated immunoassays in BioFINDER and ADNI cohorts. Alzheimers Dement. 2018;14:1470-81.

11. Schindler SE, Gray JD, Gordon BA, Xiong C, Batrla-Utermann R, Quan M, et al. Cerebrospinal fluid biomarkers measured by Elecsys assays compared to amyloid imaging. Alzheimers Dement. 2018;14:1460-9.

12. Shaw LM, Blennow K, Buck K, Eichenlaub U, Lifke V, Widmann M, et al. Analysis of cerebrospinal fluid (CSF) biomarkers to predict risk of clinical decline and progression to dementia in patients with mild cognitive impairment and mild cognitive symptoms. Alzheimers Dement. 2018;14(Suppl):P1178-9.

13. Dubois B, Feldman HH, Jacova C, Hampel H, Molinuevo JL, Blennow K, et al. Advancing research diagnostic criteria for Alzheimer's disease: the IWG-2 criteria. Lancet Neurol. 2014;13:614-29.

14. Jack CR Jr, Bennett DA, Blennow K, Carrillo MC, Dunn B, Haeberlein SB, et al. NIA-AA research framework: toward a biological definition of Alzheimer's disease. Alzheimers Dement. 2018;14:535-62.

15. Mattsson N, Lönneborg A, Boccardi M, Blennow K, Hansson O, Geneva Task Force for the Roadmap of Alzheimer's Biomarkers. Clinical validity of cerebrospinal fluid Aß42, tau, and phospho-tau as biomarkers for Alzheimer's disease in the context of a structured 5-phase development framework. Neurobiol Aging. 2017;52:196-213.

16. Ellis KA, Bush Al, Darby D, De Fazio D, Foster J, Hudson P, et al. The Australian imaging, biomarkers and lifestyle (AIBL) study of aging: methodology and baseline characteristics of 1112 individuals recruited for a longitudinal study of Alzheimer's disease. Int Psychogeriatr. 2009;21:672-87.

17. Li QX, Villemagne VL, Doecke JD, Rembach A, Sarros S, Varghese S, et al. Alzheimer's disease normative cerebrospinal fluid biomarkers validated in PET amyloid- $\beta$ characterized subjects from the Australian Imaging, Biomarkers and Lifestyle (AIBL) study. J Alzheimers Dis. 2015;48:175-87.

18. Rembach A, Evered LA, Li QX, Nash T, Vidaurre L, Fowler CJ, et al. Alzheimer's disease cerebrospinal fluid biomarkers are not influenced by gravity drip or aspiration extraction methodology. Alzheimers Res Ther. 2015;7:71.

19. Vanderstichele H, Bibl M, Engelborghs S, Le Bastard N, Lewczuk P, Molinuevo JL, et al. Standardization of preanalytical aspects of cerebrospinal fluid biomarker testing for Alzheimer's disease diagnosis: a consensus paper from the Alzheimer's Biomarkers Standardization Initiative. Alzheimers Dement. 2012;8:65-73.

20. Bittner $\mathrm{T}$, Zetterberg $\mathrm{H}$, Teunissen $\mathrm{CE}$, Ostlund RE Jr, Militello M, Andreasson $U$, et al. Technical performance of a novel, fully automated electrochemiluminescence immunoassay for the quantitation of $\beta$-amyloid (1-42) in human cerebrospinal fluid. Alzheimers Dement. 2016;12:517-26.

21. Blennow K. 2018 update on the Alzheimer's Association quality control (QC) program for CSF biomarkers. 2018. https://www.alz.org/media/Documents/ global-csf-biomarkers-july-2018-qc-program-1.pptx. Accessed 24 Sept 2019.

22. Kollmorgen $\mathrm{G}$, Teunissen $\mathrm{CE}$, Christenson RH, Powers JL, Manuilova E, Weigel $A$, et al. Multicenter evaluation of the analytical characteristics of the
Elecsys $^{\circledast}$ Total-Tau cerebrospinal fluid (CSF) and Elecsys ${ }^{\circledast}$ Phospho-Tau (181P) CSF immunoassays. Alzheimers Dement. 2018;393(Suppl):1-285.

23. Bourgeat $P$, Doré $V$, Fripp J, Ames $D$, Masters $C L$, Salvado $O$, et al. Implementing the centiloid transformation for ${ }^{11} \mathrm{C}-\mathrm{PiB}$ and $\beta$-amyloid ${ }^{18}$ F-PET tracers using CapAIBL. Neuroimage. 2018;183:387-93.

24. Rowe CC, Ellis KA, Rimajova M, Bourgeat P, Pike KE, Jones $G$, et al. Amyloid imaging results from the Australian Imaging, Biomarkers and Lifestyle (AIBL) study of aging. Neurobiol Aging. 2010;31:1275-83.

25. Rowe CC, Pejoska S, Mulligan RS, Jones G, Chan JG, Svensson S, et al. Headto-head comparison of 11C-PiB and 18F-AZD4694 (NAV4694) for $\beta$-amyloid imaging in aging and dementia. J Nucl Med. 2013;54:880-6.

26. Thurfjell L, Lilja J, Lundqvist R, Buckley C, Smith A, Vandenberghe R, et al. Automated quantification of 18F-flutemetamol PET activity for categorizing scans as negative or positive for brain amyloid: concordance with visual image reads. J Nucl Med. 2014;55:1623-8.

27. Clark CM, Schneider JA, Bedell BJ, Beach TG, Bilker WB, Mintun MA, et al. AV45-A07 study group. Use of florbetapir-PET for imaging beta-amyloid pathology. JAMA. 2011;305:275-83

28. Clark CM, Pontecorvo MJ, Beach TG, Bedell BJ, Coleman RE, Doraiswamy PM, et al. Cerebral PET with florbetapir compared with neuropathology at autopsy for detection of neuritic amyloid-beta plaques: a prospective cohort study. Lancet Neurol. 2012;11(8):669-78.

29. Thurfjell L, Lundqvist R, Buckley C, Smith A, Sherwin P. Automated quantification of [18F]flutemetamol data - comparison with standard of truth based on histopathology. J Nucl Med. 2013;54(2):302.

30. Youden WJ. Index for rating diagnostic tests. Cancer. 1950:3:32-5.

31. DeLong ER, DeLong DM, Clarke-Pearson DL. Comparing the areas under two or more correlated receiver operating characteristic curves: a nonparametric approach. Biometrics. 1988;44:837-45.

32. Morris E, Chalkidou A, Hammers A, Peacock J, Summers J, Keevil S. Diagnostic accuracy of (18)F amyloid PET tracers for the diagnosis of Alzheimer's disease: a systematic review and meta-analysis. Eur J Nucl Med Mol Imaging. 2016;43:374-85.

33. Palmqvist $\mathrm{S}$, Zetterberg $\mathrm{H}$, Mattsson $\mathrm{N}$, Johansson P, Alzheimer's Disease Neuroimaging Initiative, Minthon L, et al. Swedish BioFINDER Study Group Detailed comparison of amyloid PET and CSF biomarkers for identifying early. Alzheimer Dis Neurol. 2015;85:1240-9.

34. Wang MJ, Yi S, Han JY, Park SY, Jang JW, Chun IK, et al. Analysis of cerebrospinal fluid and [11C]PIB PET biomarkers for Alzheimer's disease with updated protocols. J Alzheimers Dis. 2016;52:1403-13.

35. Jack CR Jr, Knopman DS, Jagust WJ, Shaw LM, Aisen PS, Weiner MW, et al. Hypothetical model of dynamic biomarkers of the Alzheimer's pathological cascade. Lancet Neurol. 2010:9:119-28.

36. Fourier A, Portelius E, Zetterberg H, Blennow K, Quadrio I, Perret-Liaudet A. Pre-analytical and analytical factors influencing Alzheimer's disease cerebrospinal fluid biomarker variability. Clin Chim Acta. 2015;449:9-15.

37. Vanderstichele HM, Janelidze S, Demeyer L, Coart E, Stoops E, Herbst V, et al. Optimized standard operating procedures for the analysis of cerebrospinal fluid $A \beta 42$ and the ratios of $A \beta$ isoforms using low protein binding tubes. J Alzheimers Dis. 2016:53:1121-32.

\section{Publisher's Note}

Springer Nature remains neutral with regard to jurisdictional claims in published maps and institutional affiliations.

Ready to submit your research? Choose BMC and benefit from:

- fast, convenient online submission

- thorough peer review by experienced researchers in your field

- rapid publication on acceptance

- support for research data, including large and complex data types

- gold Open Access which fosters wider collaboration and increased citations

- maximum visibility for your research: over $100 \mathrm{M}$ website views per year

At $\mathrm{BMC}$, research is always in progress.

Learn more biomedcentral.com/submissions 